\title{
Marker-free Transgenic Rice Lines with a Defensin Gene are Potentially Active against Phytopathogenic Fungus Sarocladium oryzae
}

\author{
M. PÉREZ-BERNAL ${ }^{1 *}$, M. DELGADO ${ }^{1}$, A. CRUZ ${ }^{2}$, D. ABREU ${ }^{1}$, \\ O. VALDIVIA ${ }^{1}$ and R. ARMAS ${ }^{1}$ \\ ${ }^{1}$ Center for Genetic Engineering and Biotechnology of Sancti Spiritus P.O. Box 83, \\ P.C. 60200 , Sancti Spiritus, Cuba \\ ${ }^{2}$ National Institute of Agricultural Sciences, UCTB Los Palacios, \\ Carretera La Francia km 1 1⁄2, Pinar del Rio, Cuba
}

(Received: 8 February 2017; accepted: 7 March 2017)

\begin{abstract}
In this work it was developed marker-free transgenic indica rice plants (cv J-104) by biolistic co-transformation and segregation approach. We attempted to express the NmDef02 antifungal defensin. Primary transformants were regenerated from embryogenic callus on culture medium with $50 \mathrm{mg} / \mathrm{L}$ hygromycin. Screening of $h p t$-marker-free transgenic lines was made by PCR in $\mathrm{T}_{1}$ progeny lines, germinated on semisolid medium without hygromycin. Relative expression of $\mathrm{NmDef02}$ mRNA was examined by quantitative RT-PCR in marker-free $\mathrm{T}_{1}$ plants. In vitro antifungal test was performed by disk diffusion assay against Sarocladium oryzae.

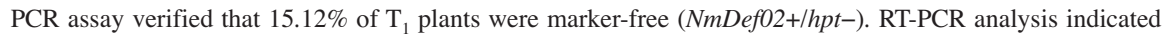
that $\mathrm{NmDef02}$ gene was successfully transcribed and the transgenic lines displayed different expression levels of the NmDef02 cDNA. Protein extracts of marker-free lines with high relative expression of $\mathrm{NmDef02}$ inhibited fungus mycelial growth around disks. In contrast, it was confirmed fungus proliferation on disks impregnated with protein extracts of non-transgenic plants. The results of the present work demonstrated that the expression of the NmDef02 defensin in transgenic rice plants is effective against the phytopathogenic fungus Sarocladium oryzae under in vitro conditions. Thus, NmDef02 defensin could be a useful tool for J-104 rice improvement.
\end{abstract}

Keywords: Antifungal activity, co-transformation, defensin, rice, Sarocladium oryzae.

Rice fungal diseases are serious agricultural problem worldwide. Fungicides are commonly used to fungus control. But this is becoming less acceptable since it increases the potential for the build-up of resistance of pathogens to fungicides, and also conflicts with the public concern for fungicide residues on human health and environment. Plant genetic engineering is then an alternative for providing resistance to fungus in rice. This approach is advantageous for introducing disease resistance into elite rice cultivars, since transgenic plants can acquire a single desired trait without any alteration of the original genetic background.

\footnotetext{
* Corresponding author; e-mail: maylin.perez@cigb.edu.cu
} 
Different genetic strategies have been used to generate disease-resistant plants, which include utilization of antimicrobial genes of both plant and non-plant origin, as well as the expression of plant disease-response pathway components (Coca et al., 2006). Defensins are a class of evolutionarily and structurally related small, highly basic, cysteine-rich peptides, displaying a broad-spectrum in vitro antifungal activity (Thomma et al., 2002).

The first attempt to evaluate of transgenic plants containing foreigner antifungal defensin genes was done in tobacco plants expressing Rs-AFP2, a peptide from radish. High levels of peptide expression were observed in the transformed tobacco plants, as well as an increasing resistance towards the phytopathogenic fungus Alternaria longipes (Terras et al., 1995). The same defensin was used for studies with transgenic apple plants and evaluation against pathogenic fungi species (De Bondt et al., 1999). Tomato lines have also been transformed with Rs-AFP2, increasing their antifungal activity against $A l$ ternaria solani, F. oxysporum, Phytophthora infestans, and Rhizoctonia solani (Parashina et al., 2000). It was demonstrated that the crude extract of tomato leaves containing the radish defensin could decrease the activity of all the fungi cited above. Jha and Chattoo (2010) transformed this peptide into rice (Oryza sativa L. cv. Pusa basmati 1). The transgenic plants were tested in vitro and in vivo against Magnaporthe oryzae and Rhizoctonia solani, the main causes of rice losses in agriculture, revealing that overexpression of Rs-AFP2 can control the rice blast and sheath blight diseases (Jha and Chatoo, 2010).

Portieles et al. (2010) reported a novel defensin gene, NmDef02, isolated from the non-cultivated wild tobacco species Nicotiana megalosiphon. Constitutive expression of NmDef02 gene in transgenic tobacco and potato plants enhanced resistance against various plant microbial pathogens, including the oomycete Phytophthora infestans, causal agent of the economically important potato late blight disease (Portieles et al., 2010).

For efficient and successful selection of transgenics, a selectable marker gene for antibiotic or herbicide resistance is incorporated into the transgenic plants. However, once a transgenic event has been selected, marker genes are undesirable: their presence in transgenic plants may raise public and regulatory concerns and may have technological disadvantages (Chong-Pérez and Angenon, 2013). Many approaches have been reported to remove selectable marker genes since the transformation technology was developed. One of the earliest methods was based on co-transformation of a transgene and a selectable marker delivered by two separate DNA molecules and thereafter, segregation of both in the progeny (Yau and Stewart, 2013).

The present study was undertaken to develop marker-free transgenic indica rice plants (cv J-104), based on the biolistic co-transformation and segregation approach. We attempted to express a synthetic version of the novel defensin gene NmDef02, reported by Portieles et al. in 2010. It was evaluated the in vitro antifungal activity of transgenic rice lines against the phytopathogenic fungus Sarocladium oryzae. 


\section{Materials and Methods}

\section{Plasmid construction}

The plasmid pA9NmDef02 contained the gene of interest, which was a synthetic version of the novel defensin gene $\mathrm{NmDef02}$ (Portieles et al., 2010), isolated from $\mathrm{Ni}$ cotiana megalosiphon. This gene was placed under the control of the A9 constitutive promoter, based on CaMV35S plus a quadruplicated Agrobacterium octopine synthase enhancer element and the first exon/intron/exon of actin 1 gene from rice. The original sequences that may affect gene expression in rice were avoided in the NmDef02, and the codons were optimized based on preferred codons in rice. During the synthesis, the original signal peptide was substituted by that of the Dahlia merckii antimicrobial peptide 1 (Dm-AMP1) which had successfully targeted to rice apoplast (Jha et al., 2009).

The plasmid p35SHyg contained the hygromycin phosphotransferase gene (hpt) as selectable marker, regulated by CaMV35S promoter.

\section{Particle bombardment and selection of transformants}

Scutellar rice calluses were bombarded with a DuPont PDS-1000/He Biolistic Delivery System. Gold particles of $1.0 \mu \mathrm{m}$ diameter were coated with the DNA plasmid as described by Taylor and Vasil (1991). In each bombardment 15 calli of 2-3 mm in length were placed onto N6 callus culture medium (Chu et al., 1975) with $0.8 \mathrm{M}$ mannitol in 6-cm diameter dishes, at $6 \mathrm{~cm}$ from stopping plate to callus tissue, and bombarded with $0.154 \mu \mathrm{g}$ gold and $0.350 \mu \mathrm{g}$ DNA, after a vacuum of $700 \mathrm{~mm} \mathrm{Hg}$ was attained with a helium pressure adjusted at 7.5. The plasmid ratio in the DNA mix was $4: 1$ of pA9NmDef02 and p35SHyg, respectively.

After bombardment, calli were kept on medium with $0.8 \mathrm{M}$ mannitol $16 \mathrm{~h}$ in the dark at $28{ }^{\circ} \mathrm{C}$. Primary transformants were selected during two weeks on N6 medium with hygromycin $50 \mathrm{mg} / \mathrm{l}$. The plant regeneration from calli was assessed using MS (Murashige and Skoog, 1962) salts and vitamins, $30 \mathrm{~g} / \mathrm{L}$ maltose, $3 \mathrm{mg} / \mathrm{L}$ kinetin, $1 \mathrm{mg} / \mathrm{L}$ NAA, 0,5 mg/L 6-BAP, hygromycin $50 \mathrm{mg} / \mathrm{l}$ and 4,5 g/L Phytagel. Cultures were maintained at $25 \pm 2{ }^{\circ} \mathrm{C}$ in environmentally controlled room under illumination of 1500 lux emitted by fluorescent tubes.

Regenerated plants were transferred to rooting medium, consisted of MS (Murashige and Skoog, 1962) salts and vitamins, $30 \mathrm{~g} / \mathrm{L}$ sucrose, hygromycin $50 \mathrm{mg} / \mathrm{l}$ and $3.0 \mathrm{~g} / \mathrm{L}$ Phytagel. The plants with a height of $10 \mathrm{~cm}$ were removed from culture medium, washed their roots, placed in pots with soil and cultured in ex vitro environment under natural conditions. The seeds obtained were used for studying the segregation of the genes in plants of $\mathrm{T}_{1}$ progeny.

\section{Screening for marker free-transgenic lines}

PCR analysis was performed to detect the regenerated plants harboring the two co-transformed genes. Plants with the two genes were transplanted to soil in a culture 
house, in order to obtain fertile seeds. The screening of marker-free lines was made by PCR in plants of $\mathrm{T}_{1}$ progeny, which were germinated in MS (Murashige and Skoog, 1962) semisolid medium without hygromycin.

Plant DNA used for PCR analysis was extracted from leaves using the protocol of Dellaporta et al. (1983). The PCR reactions were carried out in a total volume of $30 \mu 1$ including $0.5 \mu \mathrm{g}$ template DNA, $3.0 \mu 110 \mathrm{X}$ buffer, $3.0 \mu 110 \mathrm{mM}$ dNTP, $3.0 \mu 125 \mathrm{mM}$ $\mathrm{MgCl}_{2}, 3 U$ Taq DNA Polymerase (HeberZima, Havana, Cuba), $1.2 \mu \mathrm{l}$ of each primer at $25 \mu \mathrm{M}$.

DNA was denatured at $94{ }^{\circ} \mathrm{C}$ for 3 min followed by 30 amplification cycles $\left(57^{\circ} \mathrm{C}\right.$ for $1 \mathrm{~min}, 72{ }^{\circ} \mathrm{C}$ for $1.5 \mathrm{~min}, 94^{\circ} \mathrm{C}$ for $1 \mathrm{~min}$ ) and finally $10 \mathrm{~min}$ at $72{ }^{\circ} \mathrm{C}$. The amplification products were checked by agarose gel electrophoresis. The expected product sizes were $410 \mathrm{bp}$ and $630 \mathrm{bp}$, for the NmDefO2 and hpt genes, respectively.

\section{Relative gene expression of $\mathrm{NmDef02}$ defensin in transgenic rice plants}

Total RNA was extracted from four rice (30 day-old plants) marker-free lines using the RNeasy kit (Qiagen, USA), including an on-column DNAse treatment (Qiagen) according to the manufacturer's instructions. RNA was isolated using the Dynabeads mRNA Purification kit (Dynal A.S., Oslo, Norway), according to the manufacturer's instructions. The cDNA was synthesized using an oligo-(dT) primer and the Super-Script III reverse transcriptase kit (Invitrogen) according to the manufacturer's instructions.

Quantitative real-time PCR was conducted using a Rotor-Gene 3000 PCR machine (Corbett, Sydney, Australia) with the QuantiTect SYBR Green PCR kit (Qiagen). The primer sequences from $\mathrm{NmDef02}$ defensin were:

forward 5'-AAGCTTATGCGTGAGTGCAAGGCTC-3'

reverse 5'-CTGCAGTTAGCACTCGAATATAC-3'.

Real-time PCR was used to measure the relative expression of NmDef02 transcript levels, when compared to the constitutively expressed 26S rRNA gene as an endogenous control. Real-time PCR conditions were as follows: an initial $95^{\circ} \mathrm{C}$ denaturation step for $15 \mathrm{~min}$ followed by denaturation for $15 \mathrm{~s}$ at $95^{\circ} \mathrm{C}$, annealing for $30 \mathrm{~s}$ at $60{ }^{\circ} \mathrm{C}$, and extension for $30 \mathrm{~s}$ at $72{ }^{\circ} \mathrm{C}$ for 40 cycles and analyzed on the Rotor-Gene 3000 software (Corbett). All experiments were repeated twice. The lines with the highest expression of NmDef02 defensin, inferred by quantitative PCR, were selected for in vitro antifungal test.

\section{In vitro antifungal test}

Rice leaves were ground in a cold mortar with liquid nitrogen. The frozen tissue powder ( $100 \mathrm{mg}$ ) was supplied with PBS $1 \%$ and vortexed to homogeneity. The homogenate was centrifuged at $11,000 \mathrm{~g}$ for $15 \mathrm{~min}$ at $4{ }^{\circ} \mathrm{C}$ and the supernatant was used for protein quantification following Bradford (1976) and then for in vitro antifungal assays.

To determine as a preliminary way if these plants are resistant to Sarocladium oryzae, disk diffusion assays were performed. Sarocladium oryzae was cultured on potato dextrose agar (PDA) medium at $30^{\circ} \mathrm{C}$. Mycelium discs of $1.0 \mathrm{~cm}$ in diameter were placed at the centre of PDA plates $(9.0 \mathrm{~cm})$ and incubated at $30{ }^{\circ} \mathrm{C}$. As the colonies reached 
$2.5 \mathrm{~cm}$ in diameter, filter paper discs (6 mm, Whatman No. 1) were placed on the front edge of the colonies, impregnated with $250 \mathrm{mg}$ of total soluble protein extracts of transgenic lines, as well as non-transgenic plant extract, extraction buffer and a positive control of commercial fungicide Amistar ${ }^{\circledR}$. The plates were incubated in the dark at $30{ }^{\circ} \mathrm{C}$, and they were observed every day looking for the formation of inhibitory halo.

\section{Statistical analysis}

Segregation analyses of $\mathrm{NmDefO2}$ and $h p t$ genes were done using a Chi-square binomial goodness-of-fit test $(\alpha=0.05)$ in lines of $T_{1}$ progeny, taking into account the four possible patterns of transgene insertion: NmDef02(+)hpt(+), NmDef02(+)hpt(-), $N m D e f 02(-) h p t(+)$ and NmDefO2(-)hpt(-). Data were processing with the version 15 of the Statistical Package for Social Sciences.

\section{Results and Discussion}

\section{Selection of transformants}

Many of the bombarded calli were found proliferating in the first round of selection on medium containing hygromycin. The number of proliferating calli was lesser in the subsequent rounds of selection.

Shoot regeneration from hygromycin-resistant embryogenic calli was observed after 3-4 weeks of incubation on regeneration medium (Fig. 1A). Even after 3 rounds of selection, all the non-transgenic calli escaping hygromycin selection could not be eliminated because $10 \%$ of the regenerated plants died on rooting medium with hygromy-
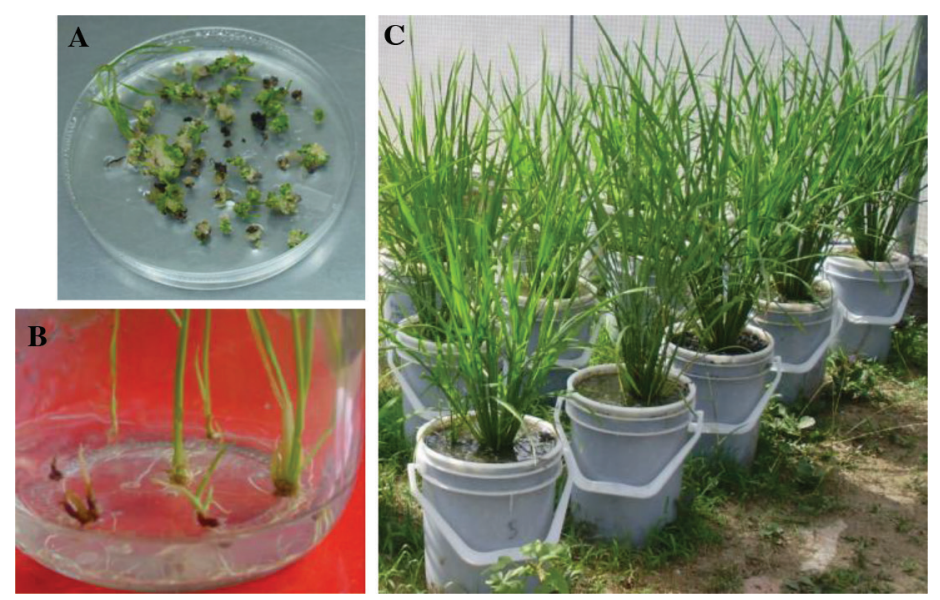

Fig. 1. (A) Shoot regeneration from hygromycin-resistant embryogenic calli 3 weeks after of incubation on regeneration medium with $50 \mathrm{mg} / \mathrm{L}$ hygromycin, (B) regenerated plants on rooting medium with 50 $\mathrm{mg} / \mathrm{L}$ hygromycin, (C) primary transformants growing under ex vitro environment 
cin (Fig. 1B). The use of selective agent in the rooting medium may reduce the number of non-transgenic plants surviving after selection rounds (Van der Vyver et al., 2013). A more extensive selection of calli on hygromycin-containing medium has not been recommended, since it seriously damages callus regeneration (Kumar et al., 2010).

Eighteen putative transformants grown successfully on rooting medium with $50 \mathrm{mg} / \mathrm{L}$ hygromycin. The integration of $\mathrm{NmDefO2}$ gene was confirmed by PCR in $72.22 \%$ and $h p t$ gene was confirmed in $100 \%$ of the plants that survived on rooting medium with hygromycin. Although the co-transformation molar ratio 4:1 favored the insertion of $\mathrm{NmDef0} 2$ over $\mathrm{hpt}$, the $100 \%$ of co-integration could be explained since hygromycin B was used as a selective agent, it favored the selection of hygromycin resistant callus and by the process; callus lines which have not the hpt gene might have got eliminated.

Co-integration of two transgenes averages $70 \%$ and greatly depends on the DNA ratio in the plasmid mixture (Chong-Pérez and Angenon, 2013). In 2010, Kumar et al. used the molar ratio 4:1 of the Bacillus thuringiensis (Bt) and hpt genes for rice co-bombardment, and $81 \%$ of the transgenic plants harbored both the transgenes. The co-integration frequency of transgenes (uidA and $h p h$ ) reported by Devi et al. (2012) was $88 \%$ in transgenic rice obtained by particle bombardment. The frequency of co-integration appears to vary in different species and also when different plasmids were used. The co-integration frequency of linked and unlinked genes can also vary between different experiments using same plasmids and also using similar experimental conditions (Devi et al., 2012).

Primary transformants, that harbored both the hpt and NmDefO2 genes, were transplanted to ex vitro environment, in cylindrical plastic pots (Fig. 1C). All lines were capable to produce healthy and fertile seed of $\mathrm{T}_{1}$ progeny three months after the transplant.

\section{Screening for marker free-transgenic lines in $T_{1}$ progeny}

Co-transformation has been reported to be an efficient and simple strategy for generation of marker-free transgenic plants. Sufficient data have been generated to believe that co-transformation followed by rounds of segregation creates marker-free plants (Wang et al., 2006; Devi et al., 2012).

The screening of marker-free transgenic lines was made by PCR in four lines of $\mathrm{T}_{1}$ progeny, which were germinated on MS (Murashige and Skoog, 1962) semisolid medium without hygromycin.

In the whole detected $\mathrm{T}_{1}$ population, PCR assays verified that $48.25 \%$ of plants

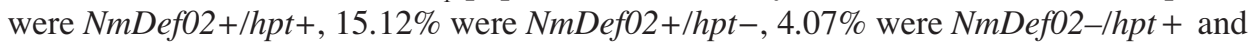

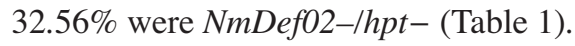

If the two foreign genes behaved as independent Mendelian loci, the expected segregation ratio in the $\mathrm{T}_{1}$ generation would be 9:3:3:1 for $\mathrm{NmDef02+/hpt+}$, NmDef02+/ hpt-, NmDef02-/hpt + and NmDef02-/hpt- events (Jiang et al., 2013). The results of binomial goodness-of-fit test $(\alpha=0.05)$ indicated that segregation proportion in analyzed lines did not coincide with Mendelian pattern.

There are reports of Mendelian segregation of co-transformed gene in rice or related species. Yao et al. (2006) co-transformed wheat (Triticum aestivum) with uidA and bar genes and obtained transgenic plants with low transgene copy number which seg- 
Table 1

Results of screening by PCR of NmDef02 and hpt genes in plants of four T1 progeny lines, which were germinated on MS semisolid medium without hygromycin

\begin{tabular}{|c|c|c|c|c|c|}
\hline Line & Category & $\begin{array}{l}\text { Plants per } \\
\text { category }\end{array}$ & $\begin{array}{c}\text { Observed } \\
\text { proportion }\end{array}$ & $\begin{array}{l}\text { Expected propor- } \\
\text { tion }\end{array}$ & $\begin{array}{l}\text { Exact significance } \\
\quad \text { (unilateral) }\end{array}$ \\
\hline \multirow[t]{4}{*}{$1-1$} & NmDef02(+)/hpt(+) & 22 & 0,550 & 0,560 & 0,510 \\
\hline & NmDef02(-)/hpt(+) & 0 & 0,000 & 0,190 & 0,000 \\
\hline & NmDef02(+)/hpt(-) & 9 & 0,230 & 0,190 & 0,345 \\
\hline & NmDef02(-)/hpt(-) & 9 & 0,230 & 0,060 & 0,001 \\
\hline \multirow[t]{4}{*}{$1-4$} & NmDef02(+)/hpt $(+)$ & 27 & 0,563 & 0,560 & 0,546 \\
\hline & NmDef02(-)/hpt(+) & 3 & 0,060 & 0,190 & 0,012 \\
\hline & NmDefO2(+)/hpt(-) & 3 & 0,060 & 0,190 & 0,012 \\
\hline & NmDef02(-)/hpt(-) & 15 & 0,310 & 0,060 & 0,000 \\
\hline \multirow[t]{4}{*}{$6-2$} & $\mathrm{NmDef0} 2(+) / h p t(+)$ & 19 & 0,442 & 0,560 & 0,080 \\
\hline & NmDef02(-)/hpt(+) & 0 & 0,000 & 0,190 & 0,000 \\
\hline & NmDef02(+)/hpt(-) & 7 & 0,016 & 0,190 & 0,412 \\
\hline & NmDef02(-)/hpt(-) & 17 & 0,400 & 0,060 & 0,000 \\
\hline \multirow[t]{4}{*}{$7-4$} & NmDef02(+)/hpt(+) & 15 & 0,366 & 0,560 & 0,010 \\
\hline & NmDef02(-)/hpt(+) & 4 & 0,100 & 0,190 & 0,088 \\
\hline & NmDef02(+)/hpt(-) & 7 & 0,170 & 0,190 & 0,471 \\
\hline & NmDef02(-)/hpt(-) & 15 & 0,370 & 0,060 & 0,000 \\
\hline
\end{tabular}

Segregation of both transgenes was achieved using categories with the four possible segregation patterns. The segregation analysis was performed by a Chi-square binomial goodness-of-fit test $(\alpha=0.05)$

regated in Mendelian form throughout three successive progenies. Kumar et al. (2010) confirmed stable inheritance of Bacillus thuringiensis (Bt) and hpt genes in indica rice according to the Mendelian (3:1) ratio.

However, Campbell et al. (2000) studied the inheritance of multiple transgenes in wheat and found ambiguous segregation ratios, evidencing that $T_{1}$ transgene analysis alone are not sufficient to properly understand genetic mechanisms involved in transgene integration and inheritance. In addition, Jiang et al. (2013) affirmed that transgene instability is a common phenomenon in modified plants from monocot species. Transgene stability is affected by multiple factors, including the structure and position of the transgene, interaction of the transgene with the endogenous genes, and its copy number. A single intact copy that integrates into the transgenic plant genome is usually desirable, as it reduces the potential for unintended insertional inactivation events (Jackson et al., 2013).

Considering the possible large-scale commercial planting, transgenic crops that do not contain selection markers are desired. The integration of the selection marker gene (hpt) and the gene of interest (NmDefO2) is crucial so that these two genes can be separated in the $\mathrm{T}_{1}$ generation. The inheritance of foreign alleles should be assessed in subsequent generations that are stable and homozygous. 


\section{Relative expression of $\mathrm{NmDef02}$ and in vitro antifungal test in marker-free $T_{1}$ plants}

The relative expression of $\mathrm{NmDef02}$ mRNA was examined by quantitative RT-PCR in four marker-free $\mathrm{T}_{1}$ plants (NmDef02+/hpt-). RT-PCR analysis indicated that NmDef02 gene was inserted in rice genomic DNA and successfully transcribed. As shown in Fig. 2, the transgenic lines displayed different expression levels of the NmDef02 cDNA, and they were significantly different $(\alpha=0.05)$ in respect to untransformed control. The variation in the NmDef02 expression might be because of position effects, the effects of transgene copy number and/or silencing processes (Portieles et al., 2010).

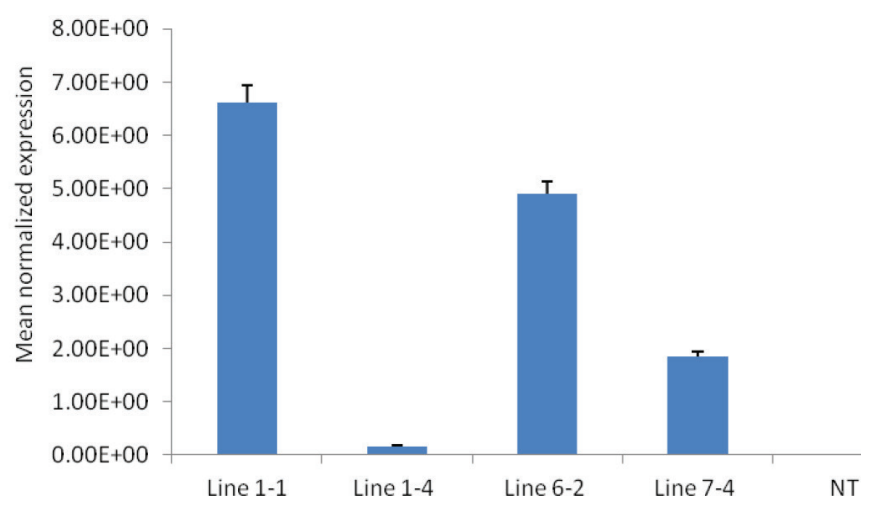

Fig. 2. Relative expression of $\mathrm{NmDef0} 2$ defensin gene in marker free transgenic rice lines of $\mathrm{T}_{1}$ progeny.

Bars represent mean values and standard error of the results obtained from three replicates. Real-time PCR was used to measure the relative expression of transcript level of NmDef02 defensin gene, when compared to the constitutively expressed $26 \mathrm{~S}$ rRNA gene as endogenous control

Two marker-free lines with high relative expression of NmDef02, 1-1 and 6-2, were chosen to develop the in vitro antifungal test against Sarocladium oryzae. Inhibitory effect of transgenic plant extracts on growth of $S$. oryzae was detected as clear inhibitory zones between growing mycelium and extract-containing filter paper discs (Fig. 3A, B). The inhibitory zones were typically formed 2 days after treatment. Microscopic observation also confirmed that the mycelia of the fungus were distorted or contracted, like in the positive control, which showed high inhibition against the fungus (Fig. 3E).

The extract of non-transformed plants was used as negative control, and it showed no zone of inhibition. This result demonstrated that the presence of rich variety of secondary metabolites in the stems and leafs of the plants did not avoid the growth of fungus (Fig. 3C). The other negative control, the protein extraction buffer, did not show inhibitory activity against the fungus growth (Fig. 3D).

Plant defensins possess potent and broad-spectrum growth-inhibitory activity against fungi. Jha and Chattoo (2010) obtained transgenic rice plants (cv. Pusa basmati 1) overexpressing the Rs-AFP2 defensin gene from Raphanus sativus, and observed that constitutive expression of Rs-AFP2 suppresses the growth of Magnaporthe oryzae and 

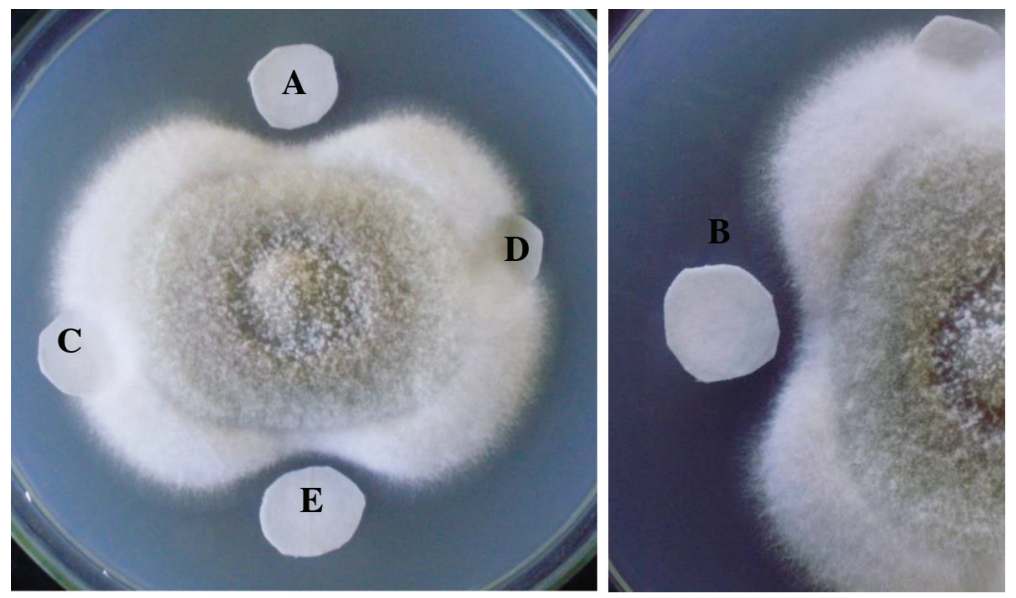

Fig. 3. In vitro antifungal test by disk diffusion assay against Sarocladium oryzae. Paper discs

( $6 \mathrm{~mm}$, Whatman No. 1) were placed on the front edge of the colonies, impregnated with (A) $250 \mathrm{mg}$ of total soluble protein extracts of transgenic line 1-1, (B) $250 \mathrm{mg}$ of total soluble protein extracts of transgenic line 6-2, (C) non-transgenic plant protein extract, (D) protein extraction buffer and (E) positive control of commercial fungicide Amistar ${ }^{\circledR}$

Rhizoctonia solani. This defensin gene was chosen in view of earlier reports that Rs-AFP2 shows in vitro antifungal activity against the fungal pathogens (Terras et al., 1995).

Portieles et al. (2010) reported that the recombinant NmDef02 defensin, produced in Pichia pastoris, exhibited antimicrobial activity against several important common plant pathogens: P. infestans, P. parasitica var. nicotianae, A. solani, F. oxysporum var. cubense and V. dahliae.

The results of the present work demonstrated that the expression of the NmDef02 defensin in transgenic rice plants is effective against the phytopathogenic fungus Sarocladium oryzae under in vitro conditions. Thus, $\mathrm{NmDef02}$ defensin could be a useful tool for J-104 rice improvement, in order to solve a serious agricultural problem, like rice fungal diseases, and to avoid the use of fungicides, which are severe threatening for human health and environment.

\section{Literature}

Bradford, M. M. (1976): A rapid and sensitive method for the quantitation of microgram quantities of protein utilizing the principle of protein-dye binding. Analytical Biochemistry 72, 248-254.

Campbell, B. T., Baenziger, P. S., Mitra, A., Soto, S. and Clemente, T. (2000): Inheritance of multiple transgenic in wheat. Crop Science 40, 1133-1141.

Chong-Pérez, B. and Angenon, G. (2013): Strategies for generating marker-free transgenic plants. In: I. SitholeNiang (ed.): Genetic Engineering. InTech., pp. 17-48.

Chu, C. C., Wang, C. C., Sun, C. S., Chen, H., Yin, K. C. and Chu, C. Y. (1975): Establishment of an efficient medium for anther culture of rice through comparative experiments on the nitrogen sources. Scientia Sinica 18, 659-668. 
Coca, M., Peñas, M., Gómez, J., Campo, S., Bortolotti, C., Messeguer, J. and San Segundo, B. (2006): Enhanced resistance to the rice blast fungus Magnaporthe grisea conferred by expression of a cecropin A gene in transgenic rice. Planta 223, 392-406.

De Bondt, A., Zaman, S., Broekaert, W., Cammue, B. and Keulemans, J. (1999): Genetic transformation of apple (Malus pumila Mill.) for increased fungal resistance: in vitro antifungal activity in protein extracts of transgenic apple expressing Rs-AFP2 or Ace-AMP1. Acta Horticulturae 484, 565-570.

Dellaporta, S. L., Wood, J. and Hicks, J. B. (1983): A plant DNA minipreparation: Version II. Plant Molecular Biology Reporter 1, 19-21.

Devi, S., Mishra, M. K. and Elliott, M. (2012): Regeneration of transgenic rice with bacterial ipt gene driven by senescence specific (SAG12) promoter by particle bombardment. Tropical Life Sciences Research 23, 39-48.

Jackson, M. A., Anderson, D. J. and Birch, R. G. (2013): Comparison of Agrobacterium and particle bombardment using whole plasmid or minimal cassette for production of high-expressing, low-copy transgenic plants. Transgenic Research 22, 143-151.

Jha, S. and Chattoo, B. B. (2010): Expression of a plant defensin in rice confers resistance to fungal phytopathogens. Transgenic Research 19, 373-384.

Jha, S., Tank, H. G., Prasad, B. D. and Chattoo, B. B. (2009): Expression of Dm-AMP1 in rice confers resistance to Magnaporthe oryzae and Rhizoctonia solani. Transgenic Research 18, 59-69.

Jiang, Y., Sun, L., Jiang, M., Li, K., Song, Y. and Zhu, C. (2013): Production of marker-free and RSV-resistant transgenic rie using a twin T-DNA system and RNAi. J. Biosciences 38, 573-581.

Kumar, S., Arul, L. and Talwar, D. (2010): Generation of marker-free Bt transgenic indica rice and evaluation of its yellow stem borer resistance. J. Appl. Genetics 51, 243-257.

Murashige, T. and Skoog, F. (1962): A revised medium for rapid growth and bioassays with tobacco tissue cultures. Physiologia Plantarum 15, 473-497.

Parashina, E. V., Serdobinski, L., Kalle, E., Lavrova, N., Avetisov, V. and Lunin, V. (2000): Genetic engineering of oilseed rape and tomato plants expressing a radish defensin gene. Russian J. Plant Physiol. 47, 417-423.

Portieles, R., Ayra, C., Gonzalez, E., Gallo, A., Rodriguez, R., Chacon, O., Lopez, Y., Rodriguez, M., Castillo, J., Pujol, M., Enriquez, G., Borroto, C., Trujillo, L., Thomma, B. and Borras, O. (2010): NmDef02, a novel antimicrobial gene isolated from Nicotiana megalosiphon confers high-level pathogen resistance under greenhouse and field conditions. Plant Biotechnology 8, 678-690.

Taylor, M. G. and Vasil, I. K. (1991): Histology of and physical factors affecting transient GUS expression in pearl millet (Pennisetum glaucum L.) embryos following microprojectile bombardment. Plant Cell Report 10, 120-125.

Terras, F. R. G., Eggermont, K., Kovaleva, V., Raikel, N., Osborn, R. and Kester, A. (1995): Small cysteine-rich antifungal proteins from radish: their role in host defense. Plant Cell. 7, 573-588.

Thomma, B., Cammue, B. and Thevissen, K. (2002): Plant defensins. Planta 216, 193-202.

Van der Vyver, C., Conradie, T., Kossmann, J. and Lloyd, J. (2013): In vitro selection of transgenic sugarcane callus utilizing a plant gene encoding a mutant form of acetolactate synthase. In Vitro Cellular and Developmental Biology 49, 198-206.

Wang, D., Zhao, Q., Zhu, D., Ao, G. and Yu, J. (2006): Particle-bombardment-mediated co-transformation of maize with a lysine rich protein gene (sb401) from potato. Euphytica 150, 75-85.

Yao, Q., Cong, 1., Chang, J. L., Li, K. X., Yang, G. X. and He, G. Y. (2006): Low copy number gene transfer and stable expression in a commercial wheat cultivar via particle bombardment. J. Experimental Botany 57, 3737-3746.

Yau, Y. Y. and Stewart, C. N. (2013): Less is more: strategies to remove marker genes from transgenic plants. BMC Biotechnology 13, 36-59. 